\title{
PALINOLOGIA DA FORMAÇÃO PIAUÍ, PENSILVANIANO DA BACIA DO PARNAÍBA: BIOCRONOESTRATIGRAFIA DE INTERVALO SELECIONADO DO POÇO 1-UN-09-PI (CAXIAS, MA, BRASIL)
}

\author{
PAULO ALVES DE SOUZA \\ Laboratório de Palinologia, DPE, IGeo, Programa de Pós-graduação em Geociências, UFRGS, Av. Bento Gonçalves, 9500, \\ 91540-000, Porto Alegre, RS, Brasil.paulo.alves.souza@ufrgs.br \\ LUCAS THETINSKI MATZEMBACHER \\ Laboratório de Palinologia, DPE, IGeo, UFRGS, Av. Bento Gonçalves, 9500, 91540-000, Porto Alegre, RS, Brasil. \\ lucas.matzembacher@gmail.com
}

MARINAABELHA \& LEONARDOBORGHI

Laboratório de Geologia Sedimentar, CCMN, IGeo, UFRJ, Av. Athos da Silveira Ramos, 274, 21941-916,

Rio de Janeiro, RJ, Brasil.marinabelha@gmail.com,lborghi@geologia.ufrj.br

\begin{abstract}
PALYNOLOGY OF THE PIAUÍ FORMATION, PENNSYLVANIAN OF THE PARNAÍBA BASIN: BIOCHRONOSTRATIGRAPHY OF A SELECTED INTERVAL WITHIN THE BOREHOLE 1-UN-09-PI (CAXIAS, MA, BRAZIL). The Pennsylvanian Piauí Formation, basal unit of the Balsas Group, is constituted by sandstones, shales and limestones, with scarce palynological records. This unit is interpreted mainly as continental to shorefacies in origin. This paper presents the palynostratigraphical results obtained for the interval between $110.60 \mathrm{~m}$ and $178.12 \mathrm{~m}$ of the borehole 1-UN-09-PI, located in Caxias, Maranhão State, which corresponds to the upper microclastic portion of this unit in the borehole. Most part of the twelve core samples studied furnished palynological associations. Among the continental palynomorphs, saccate pollen grains are dominant, mainly taeniate bisaccate and non tateniate monosaccate species. Taking into account that there is no available palynological zonation of the Pennsylvanian-Permian interval of this basin, the scheme erected for the neighboring Amazonas Basin was used for comparisons. The records of certain species from spores and pollen, such as Raistrickia cephalata Playford \& Dino, Protohaploxypinus amplus (Balme \& Hennelly) Hart, Lunatisporites onerosus Playford \& Dino, Verticipollenites sp., Striatopodocarpites sp. and Meristocorpus explicatus Playford \& Dino, allow the correlation of most of the studied interval with the Raistrickia cephalata Zone, which was recognized for the middle portion of the Nova Olinda Formation (Amazonas Basin), regarded as Pennsylvanian (Moscovian) in age.
\end{abstract}

Key words: palynology, biostratigraphy, Pennsylvanian, Piauí Formation, Parnaíba Basin.

RESUMO - A Formação Piauí, unidade pensilvaniana basal do Grupo Balsas, é considerada predominantemente de origem continental a litorânea, sendo constituída por arenitos, folhelhos e calcários, com escassos registros palinológicos. Este trabalho objetiva apresentar os resultados palinoestratigráficos obtidos da análise do intervalo entre 110,60 m e $178,12 \mathrm{~m}$ do poço 1-UN-09-PI, perfurado em Caxias, Estado do Maranhão, que corresponde à parte superior, microclástica, da unidade nesse poço. Um total de doze amostras selecionadas de testemunhos de sondagem foi estudado, cuja maioria mostrou-se palinologicamente fértil. Dentre os palinomorfos de origem continental, predominam os grãos de pólen sacados, com destaque aos bissacados teniados e monossacados não-teniados. Em virtude da ausência de zoneamento palinoestratigráfico para a seção na bacia, o zoneamento da vizinha bacia do Amazonas foi utilizado para comparação. A presença de determinadas espécies de esporos e grãos de pólen, tais como Raistrickia cephalata Playford \& Dino, Protohaploxypinus amplus (Balme \& Hennelly) Hart, Lunatisporites onerosus Playford \& Dino, Verticipollenites sp., Striatopodocarpites sp. e Meristocorpus explicatus Playford \& Dino, permite correlação da maior parte do intervalo analisado com a Zona Raistrickia cephalata, definida na porção média da Formação Nova Olinda (bacia do Amazonas), posicionada no Pensilvaniano (Moscoviano).

Palavras-chave: palinologia, bioestratigrafia, Pensilvaniano, Formação Piauí, bacia do Parnaíba.

\section{INTRODUÇÃO}

A análise estratigráfica das bacias intracratônicas brasileiras tem sido apoiada por dados bioestratigráficos, os quais são predominantemente advindos do estudo de microfósseis, especialmente palinomorfos e foraminíferos. Dentre essas bacias, a bacia do Parnaíba (sensu stricto) é a menos estudada do ponto de vista micropaleontológico, embora ocupe 
vasta área, com espessos depósitos sedimentares que testemunham distintos eventos geológicos vinculados à evolução da porção norte do Gondwana Ocidental. Para a seção relativa ao Pensilvaniano e Permiano da bacia, que corresponde litoestratigraficamente ao Grupo Balsas, os dados palinológicos são pontuais e não configuram um zoneamento bioestratigráfico.

Propostas palinoestratigráficas foram realizadas para as seções pensilvanianas e permianas das demais bacias intracratônicas brasileiras, com destaque às bacias do Amazonas (Playford \& Dino, 2000a,b) e do Paraná (Souza \& Marques-Toigo, 2003, 2005; Souza, 2006). Para a bacia do Parnaíba, os dados são precários e, por vezes, são utilizadas, por correlação, as zonas erigidas para a bacia do Amazonas, na falta de zoneamento próprio. A aplicabilidade do estudo palinológico nesta bacia é relevante devido à necessidade de correlações mais refinadas com as bacias adjacentes, visando um melhor entendimento das relações estratigráficas (laterais e horizontais) de seus depósitos.

Neste trabalho são divulgados os resultados palinoestratigráficos inéditos obtidos da análise do intervalo entre 110,60 m e 178,12 m do poço 1-UN-09-PI, na localidade de Caxias, Estado do Maranhão, e que corresponde à parte superior, microclástica, da Formação Piauí nesse poço. O único trabalho palinológico disponível para a unidade foi desenvolvido por Dino \& Playford (2002), advindo de três amostras de subsuperfície do poço 1-UN-23-PI, perfurado no município de Antônio Almeida, Estado do Piauí. O presente estudo visa ampliar os dados palinológicos para a Formação Piauí, apresentando associações ligeiramente mais jovens que as registradas por esses últimos autores, de modo a aprimorar o conhecimento da sucessão palinológica pensilvaniana da bacia. Dessa forma, prioriza-se, nesta primeira contribuição, a apresentação dos resultados de caráter biocronoestratigráfico, conforme preliminarmente apresentado em Souza et al. (2008). A lista completa de táxons identificados, com descrições e observações taxonômicas e a interpretação paleoambiental dos níveis estudados no poço compõem partes conseguintes deste trabalho.

\section{MATERIAL E MÉTODOS}

Um total de 12 níveis pelíticos do poço 1-UN-09-PI foi amostrado (Figuras 1-3), perfurado na Fazenda Brejinho, mu-

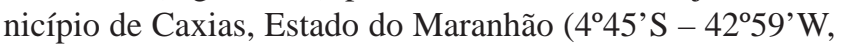
Figura 1). As amostras foram coletadas do intervalo entre 110,60 m e 178,12 m de profundidade, processadas no Laboratório de Palinologia Marleni Marques Toigo do Departamento de Paleontologia e Estratigrafia do Instituto de Geociências da Universidade Federal do Rio Grande do Sul, conforme tratamento tradicional para amostras paleozóicas (Quadros \& Melo, 1987), que consiste na desagregação física em almofariz e posterior dissolução dos componentes minerais com ataque ácido (HF, $\mathrm{HCl}$ ). Os resíduos orgânicos foram concentrados na fração entre 20 e $250 \mu \mathrm{m}$, resultando em lâminas palinológicas, as quais foram depositadas na palinoteca do citado departamento, sob codificação "MP-
P": 5969 a 5980. A identificação taxonômica e fotodocumentação das espécies foram realizadas sob microscopia óptica, utilizando-se os equipamentos Olympus CX-31 (C-330, 12.0 Megapixels) e BX-61, com tratamento nos programas Corel Photo Paint e Corel Draw (versão 12).

Para a análise biocronoestratigráfica, na ausência de um zoneamento para o intervalo Pensilvaniano-Permiano da bacia, utilizou-se o zoneamento da vizinha bacia do Amazonas, proposto por Playford \& Dino (2000b), além de informações adicionais advindas de trabalhos mais pontuais com base em fusulinídeos e conodontes.

\section{CONTEXTO GEOLÓGICO E PALEONTOLÓGICO}

O poço 1-UN-09-PI (Figuras 1,3) foi perfurado no município de Caxias, Estado do Maranhão, borda leste da bacia do Parnaíba, em trabalho vinculado ao Projeto "Carvão da Bacia do Parnaíba" (Leite et al., 1975), desenvolvido pelo Departamento Nacional de Produção Mineral e a Companhia de Recursos Minerais (DNPM/CPRM).

A maior parte da bacia do Parnaíba, que em trabalhos geológicos mais antigos é referida como "bacia do Maranhão" ou "Piauí-Maranhão", situa-se na região noroeste do nordeste brasileiro, recobrindo áreas dos estados do Maranhão e Piauí, além de parcelas dos estados do Tocantins, Pará e Ceará, numa extensão de cerca de $6.10^{5} \mathrm{~km}^{2}$ (Vaz et al., 2007). A dificuldade de compreensão do quadro tectono-sedimentar, no contexto de uma bacia única, conduziu à sua divisão em sub-bacias menores: Parnaíba (Siluriano-Triássico), Alpercatas (Jurássico-Eocretáceo), Grajaú/São Luis (Cretáceo) e Espigão Médio (Cretáceo) (Góes, 1995; Góes \& Coimbra, 1996; Góes \& Rossetti, 2001). Essas subdivisões refletem o caráter policíclico da evolução da "Província Sedimentar do Meio-Norte", designação adotada por Góes (1995) para a área equivalente à clássica denominação da "bacia do Parnaíba", uma vez que delimitam sub-bacias com gênese, estilo tectônico, preenchimento sedimentar e idades distintas. Nesse trabalho, adota-se a nomenclatura e a concepção estratigráfica apresentadas por Vaz et al. (2007), que tratam todos os depósitos sedimentares e rochas magmáticas associadas num único contexto.

A despeito de sua excelente exposição, a bacia do Parnaíba (sensu stricto) é de todas as bacias intracratônicas, aquela com menor número de dados de subsuperfície. O conhecimento estratigráfico dessa bacia é baseado no estudo da faixa aflorante, que guarda excelentes exposições, e de dados de subsuperfície, como resultado do interesse das companhias de exploração e pesquisa do país.

De acordo com Vaz et al. (2007), a sucessão de rochas sedimentares e magmáticas que compõe a bacia está disposta em cinco supersequências que, em seu depocentro, atinge a ordem de $3.500 \mathrm{~m}$ de espessura. Da base para o topo, as unidades foram denominadas como "Supersequência Siluriana" (litoestratigraficamente correspondente ao Grupo Serra Grande), "Supersequência MesodevonianaEocarbonífera" (Grupo Canindé), "Supersequência Neocarbonífera-Triássica" (Grupo Balsas), "Supersequência 
Jurássica" (Formação Pastos Bons), "Supersequência Cretácea" (formações Codó, Corda, Grajaú e Itapecuru). Além disso, as rochas ígneas intrusivas (diques e soleiras) e extrusivas presentes, de composição básica, foram divididas em duas unidades, Formação Mosquito (Neotriássico/ Eojurássico) e Formação Sardinha (Eocretáceo).

O Grupo Balsas é composto pelas formações Piauí, Pedra de Fogo, Motuca e Sambaíba (Figuras 1-3). A Formação Piauí corresponde a depósitos continentais e litorâneos, sob condições de severa aridez, representados por arenitos, folhelhos e calcários (Góes \& Feijó, 1994), e posicionados no Pensilvaniano por Daemon (1974), Campanha \& Rocha-Campos (1979) e Dino \& Playford (2002), com base em palinologia e conodontes. A Formação Pedra de Fogo apresenta duas seqüências de arenitos, que representam depósitos de dunas (intervalo inferior) e ambiente litorâneo (superior), com presença localizada de biostromas com estromatólitos, depositados em planície de maré. Com base em dados palinológicos, determinados níveis da unidade (Membro Trisidela) foram posicionados no Permiano Superior (Dino $e t$ al., 2002). A Formação Motuca consiste de folhelhos vermelhos, com níveis de siltito, localmente com estromatólitos dômicos, representando deposição em ambiente lacustre ou lagunar, do Permiano Superior/Triássico Inferior (Góes \& Feijó, 1994; Vaz et al., 2007). No topo da supersequência, a Formação Sambaíba (Triássico) é concernente a arenitos finos, caolínicos, com granulometria bimodal, interpretados como eólicos.
Lima \& Leite (1978) dividiram a Formação Piauí em duas sucessões. A inferior é composta de arenitos cor-de-rosa, médios, maciços ou com estratificação cruzada de grande porte e intercalações de folhelhos vermelhos, enquanto que a superior é constituída de arenitos vermelhos, amarelos, finos a médios, com intercalações de folhelhos vermelhos, calcários e finas camadas de sílex. Siltitos e lentes conglomeráticas também ocorrem (Caputo, 1984). Para os autores, a unidade corresponderia a ambientes fluviais, com contribuição eólica e breves incursões marinhas, num clima semi-árido a desértico.

A Supersequência Neocarbonífera-Triássica da bacia documenta fósseis de natureza variada, alguns dos quais utilizados para fins paleoambientais e bioestratigráficos. Restos de macrofitofósseis (Calamites e Pecopteris) ocorrem nas camadas inferiores da Formação Piauí, enquanto braquiópodes, bivalves, gastrópodes, cefalópodes, trilobitas e conodontes ocorrem nos níveis mais superiores (síntese em Petri \& Fúlfaro, 1988). A Formação Pedra de Fogo documenta um conteúdo paleontológico relativamente diversificado, com anfíbios (Prionosucchus), restos de peixes (Ctenacanthus), pequenos bivalves, caules de samambaias arborescentes (Tietea, Psaronius), filicíneas (Grammatopteris, Dernbachia), samambaias epífitas (Botryopteris), esfenófitas arborescentes (Arthropitys) e caules de diferentes gimnospermas, conforme sintetizado em Dias-Brito et al. (2007), além de estromatólitos (Petri \& Fúlfaro, 1988).

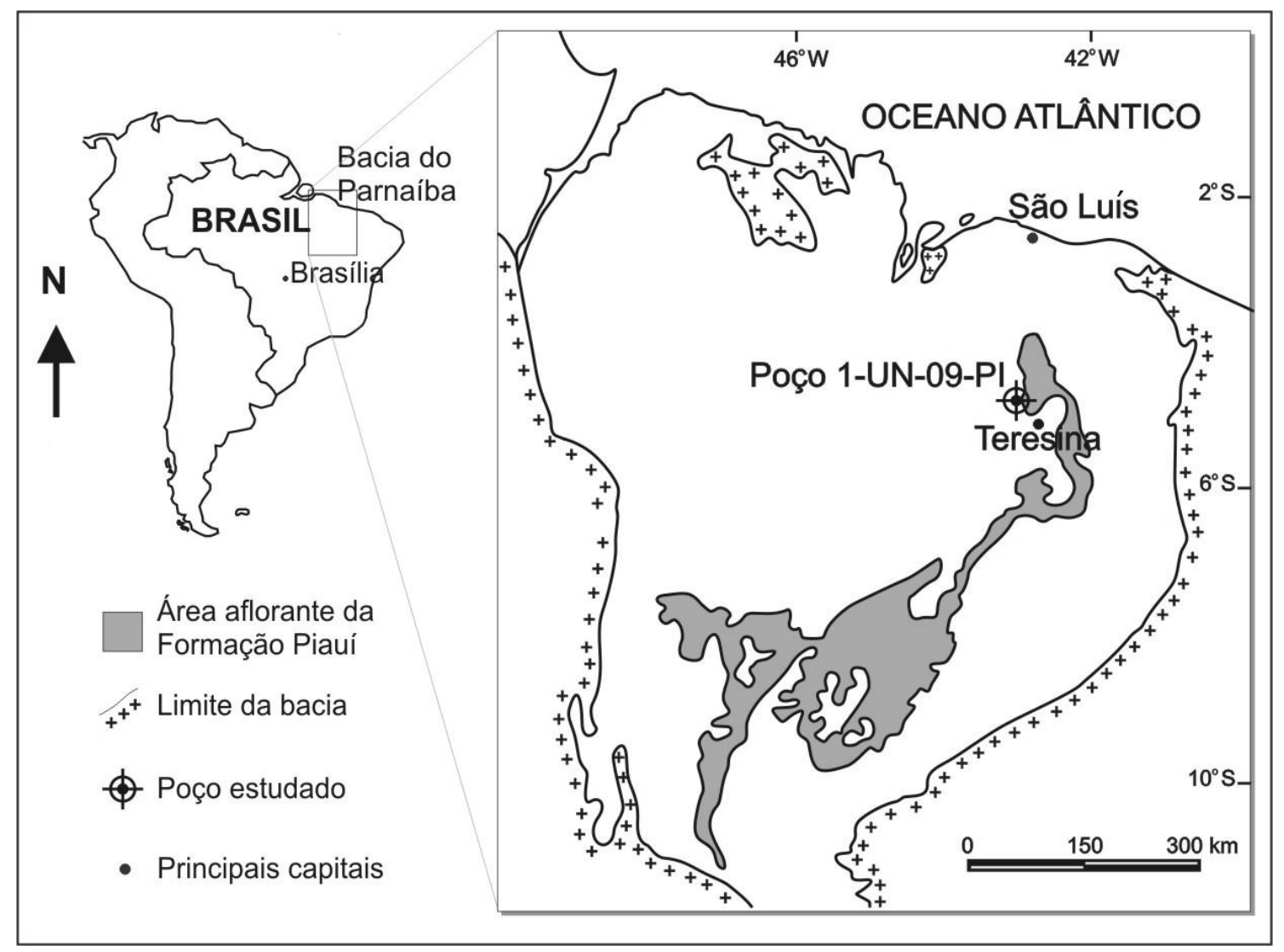

Figura 1. Área da bacia do Parnaíba, com destaque à localização do poço 1-UN-9-PI.

Figure 1. Parnaíba Basin area and location of the borehole 1-UN-09-PI. 
Alguns dos restos vegetais ocorrentes na Formação Pedra de Fogo também estão presentes na base da Formação Motuca, principalmente troncos silicificados, os quais, em Filadélfia (TO), constituem o "Monumento Natural das Árvores Fossilizadas do Tocantins" (Dias-Brito et al., 2007). Restos de peixes paleoniscídeos (Palaeoniscos, Elonichthys) e gastrópodes (Pleurotomaria) são também conhecidos na Formação Motuca, enquanto na Formação Sambaíba há somente suspeitas da presença de micrósporos (Petri \& Fúlfaro, 1988).

Dados palinológicos são relativamente escassos nas unidades do Grupo Balsas. A contribuição inédita de Müller (1962) é indisponível para consulta. Informações sobre ocorrências de fósseis, incluindo palinomorfos, são tam-

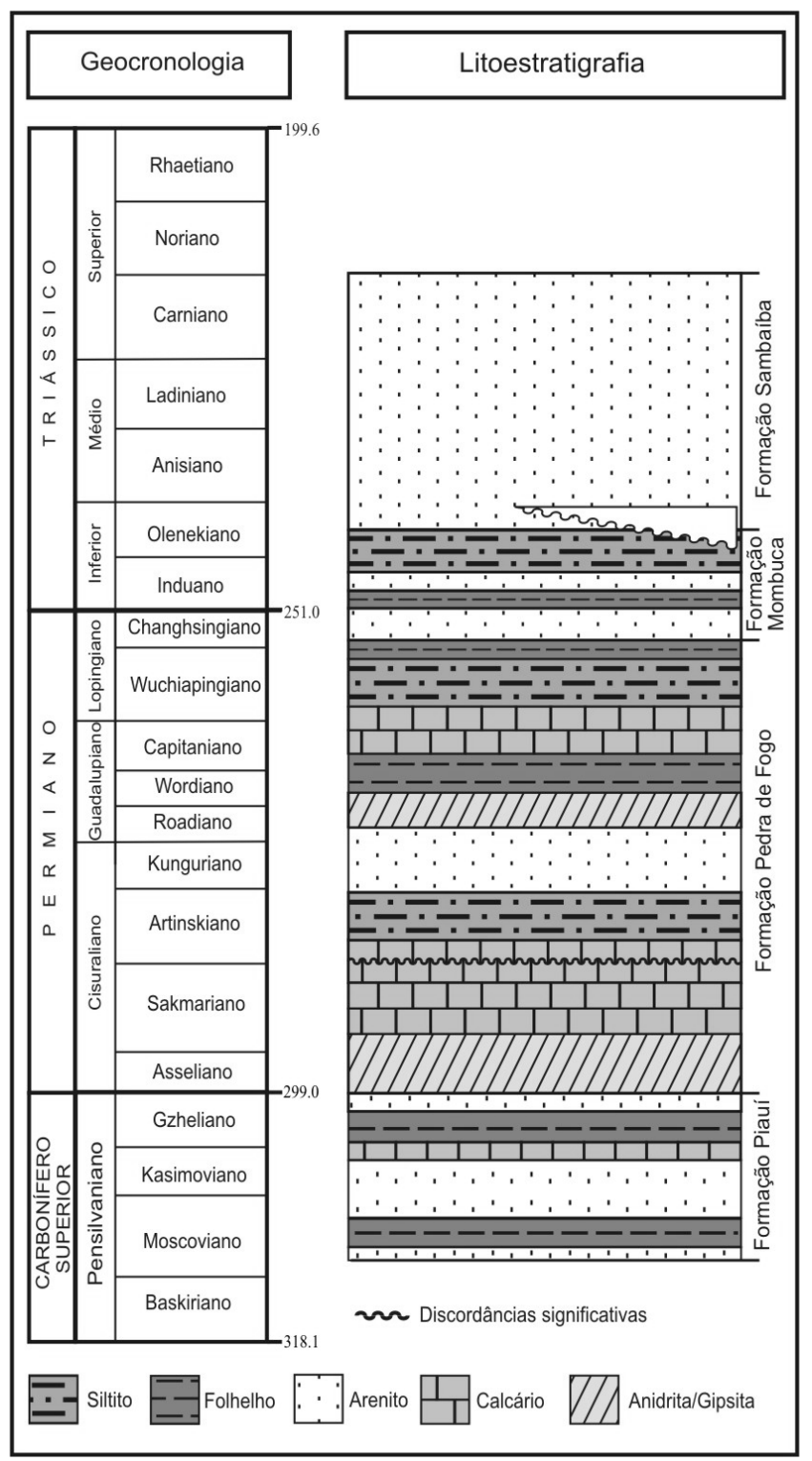

Figura 2. Coluna estratigráfica do intervalo Pensilvaniano-Triássico (Grupo Balsas) da bacia do Parnaíba (modificado de Vaz et al., 2007).

Figure 2. Stratigraphic section of the Pennsylvanian-Triassic interval (Balsas Group) of the Parnaíba Basin (modified from Vaz et al., 2007). bém encontradas em relatórios internos, tais como aqueles desenvolvidos pelo Serviço Geológico do Brasil. Bharadwaj et al. (1976) apresentaram estudos palinológicos pontuais na bacia. Contudo, trata-se de contribuição de difícil utilização para fins bioestratigráfico, devido à falta de controle estratigráfico. Para a Formação Piauí, Dino \& Playford (2002) descreveram conjuntos palinológicos registrados em três níveis da porção inferior da unidade, do poço 1-UN-23-PI, caracterizados pelo domínio de esporos cavados e pseudosacados dos gêneros Spelaeotriletes, Vallatisporites e Cristatisporites. Os autores reconheceram grande semelhança com a Zona Illinites unicus, da bacia do Amazonas (Playford \& Dino, 2000b), atribuindo idade pensilvaniana (Westphaliano) à seção estudada. Dados palinológicos são também disponíveis para a porção superior da Formação Pedra de Fogo (Dino et al., 2002), advindos de 10 amostras dos poços 1 CL-1-MA e 1-MS-1-MA. Segundo os autores, grãos de pólen teniados são os elementos dominantes das assembléias, com grande grau de similaridade com a Zona Tornopollenites toreutos, da bacia do Amazonas (Playford \& Dino, 2000b), cuja idade foi atribuída ao Permiano Superior.

As idades apresentadas na Figura 2 são baseadas em Vaz et al. (2007), não refletindo a interpretação dos presentes autores, uma vez que os dados aqui apresentados são restritos à Formação Piauí, base do Grupo Balsas. Do poço estudado, as informações paleontológicas já publicadas são restritas ao registro de grãos de pólen, acritarcos e cutículas, incluídas em relatório interno da CPRM, de cunho geral (Cruz et al., 1973), indisponível para análise.

\section{RESULTADOS}

Conjuntos palinológicos compostos por palinomorfos, fitoclastos e matéria orgânica amorfa foram recuperados de todas as 12 amostras processadas (110,60 a 178,12 m). Palinomorfos são os elementos mais abundantes e mais bem preservados no intervalo entre 144,40 e 178,12 m. Dentre os palinomorfos de origem continental, predominam os grãos de pólen sacados, com destaque aos bissacados teniados e monossacados não-teniados; esporos são subordinados. Vesículas arredondadas, de classes diferenciadas de tamanho, foram registradas em todos os níveis, algumas das quais atribuídas ao gênero Leiosphaeridia Eisenack, 1958 vinculado às Prasinophyceae, representantes do microplâncton marinho. Provavelmente correspondam a pelo menos duas novas espécies, tema de trabalho a parte, que inclui também as descrições e ilustrações de todos os táxons registrados. Nessa primeira contribuição, dentre os 29 táxons identificados (oito espécies de esporos, 19 de grãos de pólen, duas de Prasinophyceae), somente espécies selecionadas são ilustradas, priorizando aquelas de maior significado biocronoestratigráfico (Figura 4), conforme listagem do Apêndice 1. 


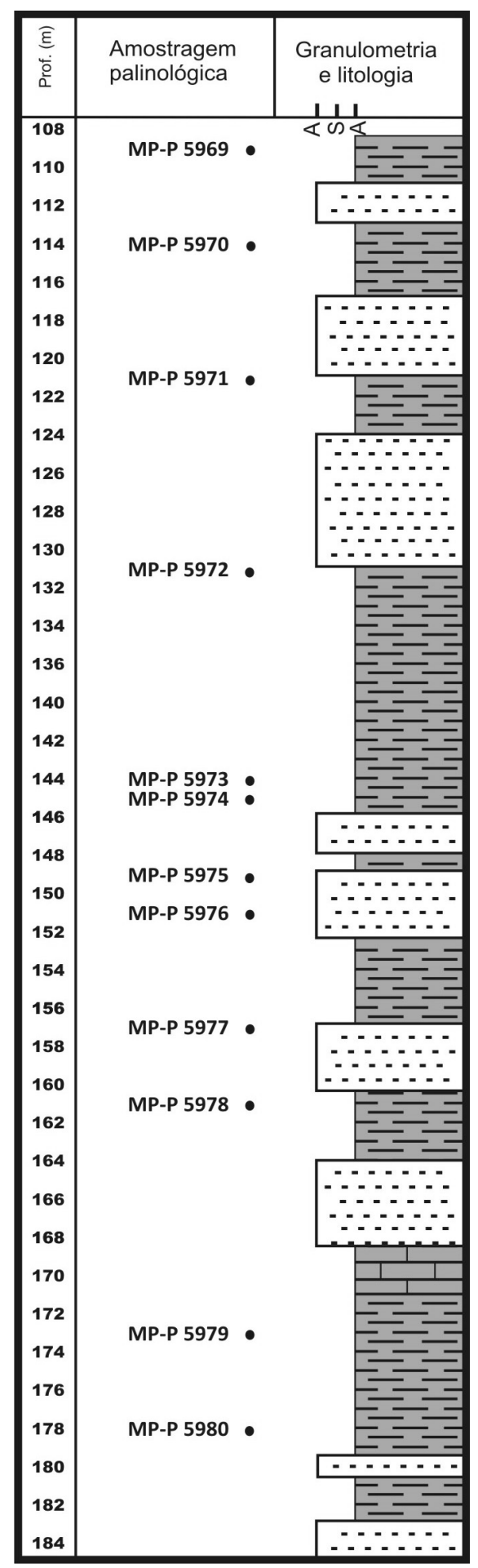

Figura 3. Perfil litológico do intervalo estudado do poço 1-UN-09$\mathrm{PI}$, com indicação da amostragem palinológica (MP-P = numeração das lâminas).

Figure 3. Lithological profile of the interval studied within the borehole 1-UN-09-PI, and palynological sampling (MP-P = slides numbers).

\section{DISCUSSÃO}

Até o momento, nenhum zoneamento palinológico foi proposto para o intervalo Pensilvaniano-Permiano da bacia do Parnaíba, em virtude, provavelmente, do menor potencial de preservação dos palinomorfos, em comparação a outras bacias intracratônicas brasileiras e, por outro lado, pelo menor investimento na perfuração de poços profundos. Amostras de afloramento são geralmente muito intemperizadas, resultando-se palinologicamente estéreis.

O zoneamento da vizinha bacia do Amazonas, proposto por Playford \& Dino (2000b), é aqui utilizado, considerando-se sua uma ampla amostragem e significativa contribuição taxonômica e bioestratigráfica. Os resultados também são comparados com os dados apresentados pontualmente na bacia do Parnaíba por Dino \& Playford (2002), para a Formação Piaú, e por Dino et al. (2002), para a Formação Pedra de Fogo. Comparações entre o material estudado com os zoneamentos estabelecidos para as bacias sul-americanas mais meridionais não são recomendadas, em razão das diferenças composicionais das associações palinológicas coetâneas como resultado das variações paleoecológicas e das posições latitudinais/longitudinais de cada área.

Correlações bioestratigráficas e idade. $O$ intervalo estratigráfico envolvendo as quatro unidades litoestratigráficas do Grupo Tapajós (formações Monte Alegre, Itaituba, Nova Olinda e Andirá) foi palinologicamente estudada por Playford \& Dino (2000a,b). Um total de sete zonas foi estabelecido, em ordem estratigráfica ascendente: Zona Spelaeotriletes triangulus, Zona Striomonosaccites incrassatus, Zona Illinites unicus, Zona Striatosporites heyleri, Zona Raistrickia cephalata, Zona Vittatina costabilis e Zona Tornopollenites toreutos. As cinco primeiras foram posicionadas no Pensilvaniano (BashkirianoGzheliano), enquanto que as duas últimas são referentes ao Permiano. Os critérios de definição das zonas são mistos, incluindo amplitude, horizontes de desaparecimento e abundância de determinados táxons.

Dentre as espécies aqui registradas, algumas são potencialmente úteis do ponto de vista bioestratigráfico. A presença de Apiculatasporites daemonii, Spelaeotriletes arenaceus, S. triangulus, Potonieisporites congoensis, Meristocorpus explicatus, Protohaploxypinus amplus e Striomonosaccites incrassatus limita o posicionamento do intervalo até o topo da Zona Raistrickia cephalata, tendo em vista que tais táxons não ocorrem em zonas mais superiores que esta.

Por outro lado, a presença Raistrickia cephalata, Verticipollenites sp. (V. sp. A em Playford \& Dino, 2000b), Striatopodocarpites sp. (S. sp. A em Playford \& Dino, 2000b) e Lunatisporites onerosus restringe o posicionamento na Zona Raistrickia cephalata, uma vez que tais táxons são confinados à citada zona, ocorrente na porção média da Formação Nova Olinda, Grupo Tapajós, bacia do Amazonas. Essas evidências indicam uma correspondência muito ajustada com o zoneamento da bacia do Amazonas, demonstrando relações genéticas entre essas bacias durante o intervalo analisado. 


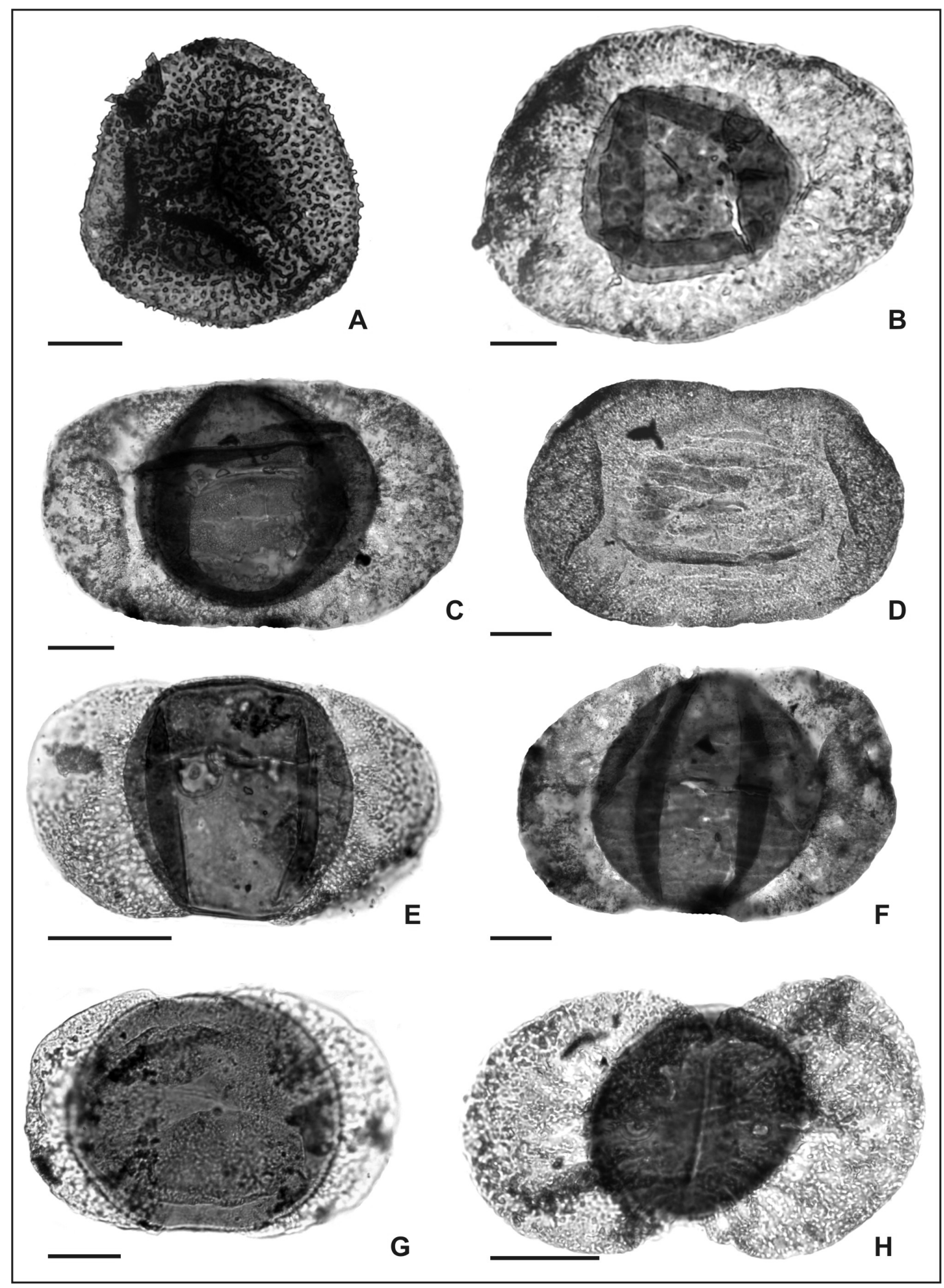

Figura 4. Fotomicrografias de espécies selecionadas do intervalo estudado (as identificações são seguidas do número de lâmina, e coordenadas England Finder). A, Spelaeotriletes arenaceus, 5974, U41/2; B, Potonieisporites congoensis, 5975, T44/3; C, Meristocorpus explicatus, 5975, O51/3; D, Striomonosaccites incrassatus, 5973, P31/3; E, Limitisporites amazonensis, 5975, T37/2; F, Protohaploxypinus amplus, 5975, M54/3; G, Lunatisporites onerosus, 5980, Q54/2; H, Verticipollenites sp., 5973, M37/2. Escalas $=20 \mu \mathrm{m}$.

Figure 4. Photomicrographs of selected species from the studied interval (identifications are followed by slides number and England Finder coordinates). Scale bars $=20 \mu \mathrm{m}$. 
A única discrepância parece ser a presença de Limitisporites amazonensis, cuja distribuição, na bacia do Amazonas, se estende até a base da Zona Vittatina costabilis. O posicionamento nessa última zona é rejeitado tendo em vista a ausência de seus elementos diagnósticos, tais como Vittatina spp., Lueckisporites spp., Hamiapollenites spp.

Embora conodontes sejam muito utilizados no estabelecimento de idades na bacia do Amazonas (vide Nascimento et al., 2005), são relativamente mais escassos na Formação Nova Olinda, onde ocorre a Zona Raistrickia cephalata. Por outro lado, fusulinídeos estão também presentes, com significativo valor biocronoestratigráfico. As associações registradas na unidade por Altiner \& Savini (1995) foram consideradas como desmoinesianas, o que indica idade entre o Moscoviano e o Kasimoviano, tendo Playford \& Dino (2000b) restringido aquela zona ao Moscoviano. Para Melo \& Loboziak (2003), os registros micropaleontológicos da Formação Monte Alegre impedem seu posicionamento em idade mais antiga que o Westphaliano, sendo mais provável no Westphaliano D, correspondente ao Moscoviano superior. Comparações com outras associações. Na bacia do Parnaíba, os únicos trabalhos disponíveis para comparações são aqueles realizados na Formação Piauí (Dino \& Playford, 2002) e na Formação Pedra de Fogo (Dino et al., 2002). Com relação ao primeiro, embora haja diversos táxons comuns, a presença de Meristocorpus ostentus Dino \& Playford, 2002 (M. sp. C em Playford \& Dino, 2000b) e Striatosporites heyleri (Doubinger) Playford \& Dino, 2000 denota equivalência com zonas suprajacentes à Zona Raistrickia cephalata. Diferenças composicionais levaram Dino \& Playford (2002) a incluir tal associação na Zona Illinites unicus, tais como a maior representação de grãos de pólen bissacados teniados após o limite inferior desta última zona. Raistrickia cephalata e Striatosporites heyleri têm suas amplitudes restritas às duas zonas imediatamente suprajacentes (Playford \& Dino, 2000b). Contudo, essas espécies foram registradas de forma escassa por aqueles autores. Esta constatação impõe cautela no uso de tais táxons como guias entre as duas bacias, embora seja compreensível alguma discrepância de uma região para outra. Com relação à associação da Formação Pedra de Fogo (Dino et al., 2002), as diferenças são mais nítidas devido à presença de táxons conhecidos e abundantes a partir de estratos permianos, tais como Vittatina spp., Lueckisporites spp. e Hamiapollenites spp.

Trabalhos gonduânicos realizados para o Pensilvaniano, aproximadamente na mesma faixa paleolatitudinal, são mais escassos e pontuais. Conjuntos palinológicos muitos semelhantes foram registrados por Azcuy et al. (2002) na Formação Tarma, bacia de Madre de Dios, Peru, compostos, predominantemente, por grãos de pólen monossacados e, subordinadamente, por grãos de pólen teniados, além de escassos esporos. A alta frequência de Illinites unicus, bem como a presença de determinadas espécies guias, conduziram os autores a correlacionar as assembléias com a Zona Illinites unicus, da bacia do Amazonas. Dentre os táxons comuns com os estudados, destacam-se espécies de
Potonieisporites, Cannanoropollis e Protohaploxypinus amplus. Illinites unicus é um dos táxons mais abundantes no material daquela bacia, o que é característico da zona epônima estabelecida na bacia do Amazonas, o que indica maior antiguidade para o material do Peru com relação à associação aqui estudada.

As associações palinológicas registradas na Formação Copacabana, bacia de Madre de Dios na Bolívia, provenientes do Poço Pando X-1 por di Pasquo (2009), têm elementos comuns com as assembléias aqui descritas, dentre os quais Meristorcopus explicatus, Lunatisporites onerosus e Verticipollenites sp. Aquelas associações foram posicionadas entre o Bashkiriano e o Moscoviano, com base em comparações com zoneamentos da Argentina e na distribuição de determinadas espécies do zoneamento do oeste europeu (Clayton et al., 1977).

\section{CONSIDERAÇÕES FINAIS}

As seguintes considerações conclusivas podem ser listadas a partir do estudo das doze amostras de testemunhos de sondagem coletadas do poço 1-UN-09-PI, que atravessou uma seção microclástica da porção superior da Formação Piauí, bacia do Parnaíba.

Diversos conjuntos palinológicos foram identificados, relativamente bem preservados e diversificados, constituídos por palinomorfos (esporos, grãos de pólen, elementos microplanctônicos), além de fitoclastos e matéria orgânica amorfa, com participação diferenciada ao longo do intervalo entre 110,60 m e 178,12 m do referido poço (maior abundância entre 144,0 e 178,12 m), com predomínio de grãos de pólen bissacados teniados. Táxons de significado biocronoestratigráfico foram identificados, dentre as 29 espécies reconhecidas.

A correlação bioestratigráfica mais aproximada é com a Zona Raistrickia cephalata, estabelecida na porção média da Formação Nova Olinda, da bacia do Amazonas, tendo como base a presença de um conjunto de espécies que limita os limites inferior e superior, desta zona. Uma idade Moscoviana superior é aqui atribuída para o intervalo estudado da Formação Piauí, tendo como base também os dados de fusulinídeos para a bacia do Amazonas. Embora algumas associações pensilvanianas da América do Sul apresentem elementos comuns correlações com unidades de bacias mais distantes são menos seguras, tendo em vista variações composicionais e amplitudes de táxons diferenciadas, como resultado de variações paleoecológicas, em distintos contextos paleolatitudinais.

A continuidade desta contribuição deverá explorar os aspectos taxonômicos, com potencial de proposição de novos táxons, além de investigar o significado paleoambiental dos conjuntos registrados, cuja preservação, abundância e diversidade justificam a intensificação do desenvolvimento de trabalhos micropaleontológicos mais sistemáticos na bacia do Parnaíba. 


\section{AGRADECIMENTOS}

Os autores agradecem ao CNPq (processo 483947/20075), pelo auxílio financeiro através de projeto de pesquisa e concessão de bolsas (PAS, LTM, LB), à ANP (PRH-18) pela concessão de bolsa (MA), ao $4^{\circ}$ Distrito do DNPM (Recife/ PE) pelo apoio e cessão de amostras, e a Alexsander L. de Souza, pelo auxílio na confecção de figuras. Contribuição apresentada no XII SBPP (Florianópolis, 02 a 05 de novembro de 2008).

\section{REFERÊNCIAS}

Altiner, D. \& Savini, R.R. 1995. Pennsylvanian foraminifera and biostratigraphy of the Amazonas and Solimões basins (north Brazil). Revue de Paléobiologie, 14:417-453.

Azcuy, C.L.; Di Pasquo, M. \& Ampuero, H.V. 2002. Late Carboniferous miospores from the Tarma Formation, Pongo de Mainique, Peru. Review of Palaeobotany and Palynology, 118:128.

Bharadwaj, D.C.; Kar, R.K. \& Navale, G.K.B. 1976. Palynostratigraphy of the Lower Gondwana deposits in Paraná and Maranhão basins, Brazil. Biological Memoirs, Paleopalynogical Serie-3, 1(1-2):53-108.

Campanha, V.A. \& Rocha-Campos, A.C. 1979. Alguns microfósseis da Formação Piauí (Neocarbonífero), Bacia do Parnaíba. Boletim do IG-USP, 10:57-67.

Caputo, M.V. 1984. Stratigraphy, tectonics, paleoclimatology and paleogeography of Northern basins of Brazil. University of California, Tese de Doutorado, $586 \mathrm{p}$.

Clayton, G.; Coquel, R.; Doubinger, J.; Gueinn, K.L.; Loboziak, S \& Owens, B. 1977. Carboniferous miospores of Western Europe: illustration and zonation. Mededelingen Rikjks Geologische Dienst, 29:1-71.

Cruz, W.B.; Aboarrage, A.M. \& Santos, M.E.C.M. 1973. Projeto carvão da Bacia do Parnaíba. Relatório de Progresso, Etapas II e III, DNPM/CPRM, Recife, 2 v.

Daemon, R.F. 1974. Palinomorfos guia do Devoniano Superior e Carbonífero Inferior das bacias do Amazonas e Parnaíba. Anais da Academia Brasileira de Ciências, 46(3/4):549-587.

Di Pasquo, M. 2009. The Pennsylvanian palynoflora of the Pando X-1 Borehole, northern Bolivia. Review of Palaeobotany and Palynology, 157(3/4):266-284.

Dias-Brito, D.; Rohn, R.; Castro, J.C.; Dias, R.R. \& Rössler, R. 2007. Floresta petrificada do Tocantins Setentrional - O mais exuberante e importante registro florístico tropical-subtropical permiano no Hemisfério Sul. In: M. Winge; C. Schobbenhaus; M. Berbert-Born; E.T. Queiroz; D.A. Campos; C.R.G. Souza \& A.C.S. Fernandes (orgs.). Sítios Geológicos e Paleontológicos do Brasil. Disponível em http://www.unb.br/ig/sigep/sitio104/ sitio104. pdf, 2007, v. 1, p. 1-15.

Dino, R. \& Playford, G. 2002. Stratigraphic and palaeoenvironmental significance of a Pennsylvanian (Upper Carboniferous) palynoflora from the Piauí Formation, Parnaíba Basin, northeastern Brazil. Paleontological Research, 6(1):2340.

Dino, R.; Antonioli, L. \& Braz, S.M.N. 2002. Palynological data from the Trisidela Member of Upper Pedra de Fogo Formation
("Upper Permian") of the Parnaíba Basin, northeastern Brazil. Revista Brasileira de Paleontologia, 3:24-35.

Góes, A.M. 1995. A Formação Poti (Carbonífero Inferior) da Bacia do Parnaíba. Programa de Pós-graduação em Geociências, Universidade de São Paulo, Tese de Doutorado, 230 p.

Góes, A.M. \& Coimbra, A.M. 1996. As bacias sedimentares da Província Sedimentar do Meio-Norte. In: SIMPÓSIO DE GEOLOGIA DA AMAZÔNIA, 5, 1996. Boletim de Resumos Expandidos, Belém, UFPA, p. 186-187.

Góes, A.M. \& Rossetti, D.F. 2001. Gênese da Bacia de São LuísGrajaú, meio-norte do Brasil. In: D.F. Rossetti; A.M. Góes, \& W. Truckenbrodt (eds.) O Cretáceo da Bacia de São LuísGrajaú, Editora MPEG, p. 15-29.

Góes, A.M. de O. \& Feijó, F.J. 1994. Bacia do Parnaíba. Boletim de Geociências da Petrobrás, 8:57-67.

Leite, J.F.; Aboarrage, A.M. \& Daemon, R.F. 1975. Projeto Carvão da Bacia do Parnaíba. Recife, DNPM/CPRM, 5 v. (relatório interno, inédito).

Lima, E. de A.M. \& Leite, J.F. 1978. Projeto estudo global dos recursos minerais da Bacia Sedimentar do Parnaíba integração geológica-metalogenética. Recife, DNPM/CPRM, $2 \mathrm{v}$.

Melo, J.H.G. \& Loboziak, S. 2003. Devonian-Early Carboniferous miospore biostratigraphy of the Amazon Basin, Northern Brazil. Review of Palaeobotany and Palynology, 124(3):131-202.

Melo, J.H.G., Loboziak, S. \& Streel, M. 1998. Latest Devonian to early Late Carboniferous biostratigraphy of Northern Brazil: an update. Bulletin du Centre de Recherches Elf Exploration Production, 22(1):13-33.

Müller, H. 1962. Report on palynological results of samples examined from wells in Maranhão. Petrobras/Rpba Relatório Interno, $44 \mathrm{p}$.

Nascimento, S.; Scomazzon, A.K.; Moutinho, L.P.; Lemos, V.B. \& Matsuda, N. 2005. Conodont biostratigraphy of the Lower Itaituba Formation (Atokan Age, Pennsylvanian), Amazonas Basin, Brazil. Revista Brasileira de Paleontologia, 8(3):193-202.

Petri, S. \& Fúlfaro, V.J. 1988. Geologia do Brasil. São Paulo, T.A. Queiroz \& EDUSP, $631 \mathrm{p}$.

Playford, G. \& Dino, R. 2000a. Palynostratigraphy of upper Palaeozoic strata (Tapajós Group), Amazonas Basin, Brazil: Part one. Palaeontographica Abteilung B, 255:1-46.

Playford, G. \& Dino, R. 2000b. Palynostratigraphy of upper Palaeozoic strata (Tapajós Group), Amazonas Basin, Brazil: Part two. Palaeontographica Abteilung B, 255:87-145.

Quadros, L.P. \& Melo, J.H.G. 1987. Método prático de preparação palinológica em sedimentos pré-mesozóicos. Boletim de Geociências da Petrobrás, 1(2):205-214.

Souza, P.A. 2006. Late Carboniferous palynostratigraphy of the Itararé Subgroup, northeastern Paraná Basin, Brazil. Review of Palaeobotany and Palynology, 138(1):9-29.

Souza, P.A. \& Marques-Toigo, M. 2003. An overview on the palynostratigraphy of the Upper Paleozoic strata of the Brazilian Paraná Basin. Revista del Museo Argentino de Ciencias Naturales, 5(2):205-214.

Souza, P.A. \& Marques-Toigo, M. 2005. Progress on the palynostratigraphy of the Permian strata in Rio Grande do 
Sul State, Paraná Basin, Brazil. Anais da Academia Brasileira de Ciências, 77(2):353-365.

Souza, P.A.; Matzembacher, L.T.; Abelha, M. \& Borghi, L. 2008. Palinologia da Formação Piauí na borda leste da Bacia do Parnaíba: dados preliminares do poço 1-UN-09-PI. In: SIMPÓSIO BRASILEIRO DE PALEOBOTÂNICA E
PALINOLOGIA, 12, 2008. Boletim de Resumos, Florianópolis, ALPP, p. 210.

Vaz, P.T.; Rezende, N.G.A.M.; Wanderley Filho, J.R. \& Travassos, W.A.S. 2007. Bacia do Parnaíba. Boletim de Geociências da Petrobrás, 15(2):253-263.

Received in January, 2009; accepted in February, 2010.

Apêndice 1. Listagem de táxons identificados neste estudo.

Appendix 1. List of taxa identified in this study.

\section{Esporos}

Anteturma Proximegerminantes R. Potonié, 1970

Turma Trilete Reinsch emend. Dettmann,1963

Suprasubturma Acavatitriletes Dettmann, 1963

Subturma Azonotriletes Luber emend. Dettmann, 1963

Infraturma Laevigati Bennie \& Kidston emend. R. Potonié, 1956

Calamospora breviradiata Kosanke, 1950

Calamospora hartungiana Schopf in Schopf, Wilson \& Bentall, 1944

Punctatisporites sp.

Infraturma Apiculati Bennie \& Kidston emend. R. Potonié, 1956

Subinfraturma Nodati Dybová \& Jachowicz, 1957

Apiculatasporites daemonii Playford \& Dino, 2000

Suinfraturma Baculati Dybová \& Jachowicz, 1957

Raistrickia cephalata Bharadwaj, Kar \& Navale, 1976

Suprasubturma Pseudosaccitriletes Richardson, 1965

Infraturma Monopseudosacciti Smith \& Butterworth, 1967

Spelaeotriletes arenaceus Neves \& Owens, 1966 (Figura 4.A)

Spelaeotriletes triangulus Neves \& Owens, 1966

Velamisporites sp.

Turma Monoletes Ibrahim, 1933

Suprasubturma Acavatomonoletes Dettmann, 1963

Subturma Azonomonoletes Luber, 1935

Infraturma Laevigatomonoleti Dybová \& Jachowicz, 1957

Laevigatosporites ovatus Wilson \& Webster, 1946

\section{Grãos de pólen}

Anteturma Variegerminantes R. Potonié, 1970

Turma Saccites Erdtman, 1947

Subturma Monosaccites Chitaley emend. R. Potonié \& Kremp, 1954

Infraturma Triletesacciti Leschik, 1955

Cannanoropollis janakii R. Potonié \& Sah, 1960

Plicatipollenites gondwanensis (Balme \& Hennelly) Lele, 1964

Plicatipollenites malabarensis (R. Potonié \& Sah) Foster, 1975

Infraturma Vesiculomonoraditi Pant, 1954

Caheniasaccites flavatus Bose \& Kar emend. Azcuy \& Di Pasquo, 2000

Costatascyclus crenatus Felix \& Burbridge emend. Urban, 1971

Crucisaccites sp.

Potonieisporites barrelis Tiwari, 1965

Potonieisporites congoensis Bose \& Maheshwari, 1968 (Figura 4B)

Potonieisporites lelei Maheshwari,1967

Potonieisporites novicus Bhardwaj emend. Poort \& Veld, 1997

Potonieisporites triangulatus Tiwari, 1965

Infraturma Striasacciti Bharadwaj, 1962

Meristocorpus explicatus Playford \& Dino, 2000 (Figura 4C)

Striomonosaccites incrassatus Playford \& Dino, 2000 (Figura 4D)

Subturma Disaccites Cookson, 1947

Infraturma Disaccitrileti Leschik, 1955

Limitisporites amazonensis Playford \& Dino, 2000 (Figura 4E)

Infraturma Striatiti Pant, 1954

Illinites unicus Kosanke emend. Jansonius \& Hills, 1976

Lunatisporites onerosus Playford \& Dino, 2000 (Figura 4G)

Protohaploxypinus amplus (Balme \& Hennelly) Hart, 1964 (Figura 4F)

Striatopodocarpites sp.

Verticipollenites sp. (Figura $4 . \mathrm{H}$ ) 
Algas clorofíceas

Divisão Prasinophyta Round, 1971

Ordem Pyramimonadales Schiller, 1925

Família Leiosphaeridaceae Sommer emend. Sommer \& Van Boekel, 1963

Leiosphaeridia spp. 